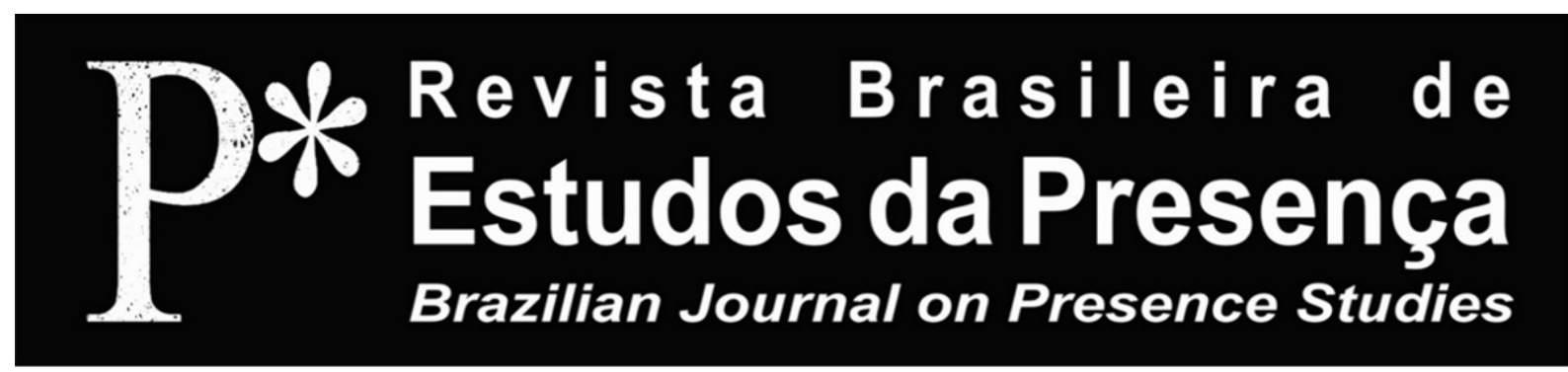

DOI - http://dx.doi.org/10.1590/2237-266043411

ISSN 2237-2660

\title{
Théâtre et Principe d'Adhésion Émergentiste
}

\section{Yannick Bressan \\ Université de Strasbourg - Strasbourg, France}

RÉSUMÉ - Théâtre et Principe d'Adbésion Émergentiste - La récente mise en évidence d'un phénomène psychologique et neurocognitif au Laboratoire d'Imagerie et de Neurosciences Cognitives (Hôpital de Strasbourg) montre combien l'alliance art et sciences peut être une voix de recherche des plus fécondes et des plus inattendues. Les applications de cette étude sur le principe d'adhésion émergentiste sont nombreuses (psycho-oncologie, art, communication...). Ainsi, il apparaît au regard des résultats obtenus et qui seront présentés brièvement dans ce texte que le théâtre, loin de n'être qu'un simple outil de divertissement, peut comme Aristote l'envisageait, participer et contribuer au bienêtre et à l'évolution de la cité. Ce texte est ainsi, à sa façon un plaidoyer pour l'interdisciplinarité active.

Mots-clés: Theatre. Neurosciences Cognitives. Psychologie. Principe d'Adhésion Émergentiste. Neuro-esthétique.

ABSTRACT - The Theater and Principle of Emergentist Adbesion - The recent Revealing of a psychological and neurocognitive phenomenon in the Laboratory of Imaging and Cognitive Neurosciences (Hospital of Strasbourg) shows us how much the alliance of art and sciences can be a fertile and an unexpected way of researching. The applications of this study on the principle of emergentist adhesion are numerous (psychology-oncology, art, communication...). So it appears with regard to the obtained results and which will be briefly presented in this text that the theater, far from being that a simple tool of entertainment can participate and to contribute, as Aristote envisaged, to the wellbeing and to the evolution of the city. This text is so, in its way a plea for the active interdisciplinarity. Keywords: Theater. Cognitive Neurosciences. Psychology. Principle of Emergentist Adhesion. Neuroaesthetics.

RESUMO - O Teatro e o Princípio de Adesáo Emergentista - A recente descoberta de um fenômeno psicológico e neurocognitivo no Laboratoire d'Imagerie et de Neurosciences Cogntivies (Hospital de Estrasburgo) mostra o quanto a aliança entre arte e ciências pode ser uma via de pesquisa das mais fecundas e inesperadas. As aplicaçóes desse estudo sobre o princípio de adesão emergentista são inúmeras (psico-oncologia, arte, comunicação). Assim, os resultados obtidos e que serão brevemente apresentados neste texto demonstram que o teatro, longe de ser somente um simples veículo de entretenimento, pode, como pensava Aristóteles, participar e contribuir ao bem estar e à evoluçáo da comunidade. Este texto constitui, da sua maneira, um pleito pela interdisciplinaridade ativa.

Palavras-chave: Teatro. Neurociências Cognitivas. Psicologia. Princípio de Adesão Emergentista. Neuroestética. 
Les neurosciences cognitives appliquées au champ de la représentation théâtrale ont récemment permis de mieux comprendre la façon dont émerge une réalité fictionnelle en lui permettant de prendre une forme d'existence effective. Une étude ${ }^{1}$ interdisciplinaire (neurosciences, esthétique, études théâtrales et psychologie cognitive) a permis de mettre en évidence en 2007-2008 le principe d'adhésion émergentiste (ou PAEm) et le lien fondamental qui unit ce principe, les évènements mis en scène et le système cognitif du spectateur.

C'est ainsi que pourra ponctuellement émerger le théâtral qui touchera émotionnellement, voire physiquement, le spectateur. Celuici perçoit un personnage en un espace-temps fictif (celui de l'histoire représentée) alors qu'il a sous les yeux un comédien sur des planches.

Comment cette substitution de réalité s'effectue-t-elle d'un point de vue neurocognitif? Quelles sont les activations cérébrales et les implications physiologiques du spectateur qui permettent à la magie du théâtral d'opérer? Quel est cet état particulier qu'en tant que spectateur nous avons tous expérimenté au moins une fois? Ce mélange de réalité et de rêve s'articulant, se juxtaposant jusqu'à ne former qu'une sensation trouble, celle d'une réalité scénique coexistant en parallèle de notre réalité proche; cette réalité scénique qui parfois, via le principe d'adhésion émergentiste peut nous entrainer dans un rire, une frayeur ou des larmes; comme si c'était vrai et bouleverser notre état psychique?

Le théâtre peut ainsi être un formidable champ expérimental in vivo pour les neurosciences cognitives. Cette première expérience de neuro-esthétique appliquée à l'étude du PAEm a dégagée des pistes expérimentales et des enjeux de réflexions des plus inattendus et des plus stimulants qui abondent en ce sens. L'approche scientifique fondamentalement systémique ${ }^{2}$ permet ainsi de poser et d'avancer sur de nombreuses questions liées à l'émergence d'une réalité.

Quelles sont précisément les activations neurologiques et l'activité physiologique lors de cette substitution de réalités? Outre les, à priori attendues, réactions physiques en réponse à l'activité scénique (comme accélération cardiaque lors d'une porte qui claque, les réactions épidermiques lors d'un moment d'angoisse ou de tendresse), qu'est-ce qui compose l'activité neurologique du spectateur lors de l'activation du principe d'adhésion émergentiste? 
En se fondant sur les découvertes misent à jour lors de cette expérience et des travaux qui suivirent, nous savons aujourd'hui comment le spectateur est appelé à une intense activité cognitive pour que s'active le PAEm afin qu'émerge la réalité théâtrale. Ne serait-ce pas là un des éléments actifs du théâtre de l'intérieur dont parle le metteur en scène Claude Régy et qui a construit l'histoire du théâtre en particulier et des représentations en général, bien avant la mimésis aristotélicienne?

Ces questions feront l'objet d'une réflexion qui nous conduira à la question centrale de notre propos: comment émerge la réalité théâtrale? En se fondant sur une étude en neuro-esthétique et sans trop entrer dans de lourds détails techniques et scientifiques, il s'agira d'aborder le phénomène psycho-cognitif qui est la pierre angulaire de notre système représentationnel et d'en comprendre les enjeux pour que la représentation théâtrale et sa réalité intrinsèque soit efficiente.

Une étude transdisciplinaire a donc été menée au Laboratoire d'Imagerie et de Neurosciences Cogntivies (LINC) avec l'Hôpital Civil de Strasbourg et le Théâtre National de Strasbourg afin de déterminer les corrélats neuronaux de l'adhésion d'un sujet-spectateur face à une représentation théâtrale. Lors de cette étude, comme bien souvent dans la recherche, des voies inattendues sont apparues aux expérimentateurs au-delà de ce qu'ils pouvaient penser découvrir.

\section{Le Lien Existentiel Unissant le Principe d'Adhésion Émergen- tiste et le Théâtral}

Lors de cette expérience nous avons relevé, et ça peut tomber sous le sens, l'adhésion comme phénomène fondamental à l'émergence d'une réalité (ici théâtrale). Par ailleurs, l'objet était de tenter de déterminer les zones cérébrales en correspondance lorsqu'un sujet accepte ou, pour paraphraser William James, a «[...] la volonté de croire» (James, 2005, p. 39) en une forme d'existence du personnage au-delà de sa vision de l'acteur. C'est l'instant (ou la suite d'instants pour être précis) où la perception de la fiction se substitue à la simple vision que le sujet-percevant a du monde qui l'entoure.

Au théâtre, lors d'une représentation, le monde qui entoure le spectateur et au sein duquel il va d'une certaine façon prendre part est un monde construit d'artificia et de quotidien. Nous assistons bien là à la mise en place d'une réalité mitoyenne, entre réalité du 
spectateur, les éléments physiques composant la réalité du sujet et la virtualité du personnage, les éléments de la fiction. Il s'agit bien d'une sorte de mitoyenneté de l'existence que cristalliserait le théâtral lors d'une représentation.

Qu'en est-il vraiment? Est-ce un phantasme de théâtreux ou une réalité neurobiologique, physiologique et cognitive? Les premiers résultats de cette étude sur le PAEm, et les travaux qui suivirent, semblent ouvrir des perspectives sur le sujet.

À ce stade de notre réflexion, deux mises en garde s'imposent. La première est sur la définition de l'adhésion telle que nous l'entendons dans principe d'adhésion émergentiste. Il ne s'agit pas ici de la définition traditionnelle d'adhésion au sens où un sujet va adhérer à une idée, une religion ou un parti politique et s'y engager. Il est entendu que cette adhésion-racine est fort probablement fondamentale dans le cadre de la mise en action neuropsychologique du PAEm mais elle n'est pas le principe d'adhésion émergentiste. Celui-ci est compris comme le phénomène psycho-cognitif qui va conduire un sujet à (se) faire émerger une réalité à laquelle il va donner plus ou moins consciemment la force de l'existence à tel point que cette réalité émergente deviendra la réalité du sujet adhérant.

Il n'est, de plus et c'est ma seconde mise au point, pas question de restreindre les résultats obtenus à un quelconque localisationnisme. Ce travail a posé les fondements premiers, philosophico-esthétiques, neurophysiologiques, psychologique et cognitifs engageant le concept d'adhésion au représenté comme substrat psychique de l'émergence d'une réalité. Il faut préciser que tout réductionnisme qui octroierait un effet neurologique à une zone précise du cerveau est exclu. Un neurodynamisme semble plus clairement en jeu entre les zones cérébrales mises au jour lors de l'expérience et l'activité physiologique observée. Il reste qu'une localisation plus précise encore des aires cérébrales en interaction lors de l'adhésion permettrait, probablement, d'aller plus loin dans la compréhension fondamentalement ontologique de l'être au monde et de notre rapport d'être humain à la réalité ainsi que de la manière dont nous (nous) la faisons émerger, par-soi $i^{3}$.

Lors de cette émergence du personnage, l'acteur, véritable «chaman» au sens d'Artaud (2001), donne vie au représenté. La virtualité du personnage semble, du moins empiriquement, glisser vers une forme d'actualité. Pour les théoriciens envisageant une part active 
du spectateur, le lieu théâtral serait donc le lieu de l'acte magique tel que l'a imaginé Antonin Artaud considérant la scène comme foyer de la vision au sein du lieu théâtral, un athanor: «Tout le théâtre est sur la scène, hors des situations et des mots» (2001, p. 102). Ainsi, la scène se fait creuset alchimique, contenant essentiel au rituel. En paraphrasant Marcel Mauss, on peut considérer que la magie théâtrale ne se définirait pas seulement «[...] par la forme de ses rites, mais par les conditions dans lesquelles ils se produisent» (1983, p. 16), donc par l'espace dévolu au spectacle.

C'est l'expérience qu'il est possible de vivre au théâtre lorsque, par exemple, on peut s'émouvoir aux larmes lors de l'assassinat du bébé Onysos (Gaudé, 2000), enfant Dieu, atrocement mutilé dans son couffin. Objectivement, quelle est la réalité de ce crime odieux? Il n'a de réalité que celle que lui prête le spectateur face au comédien porteur de l'acte. Pourtant, il est inéluctable qu'à ce moment-là, lors de cet événement théâtral, le spectateur peut être ému, horrifié, touché jusqu'au plus profond de lui. Il est touché comme si $i^{4}$ la fiction avait une présence réelle, une charge physique et psychique, pouvant affecter sa réalité proche.

Lorsque le metteur en scène est intervenu lors des répétitions dans la narration théâtrale qui a ensuite été représentée par le comédien et tous les artifices scénico-théâtraux, face au sujet-spectateur, les expérimentateurs ont observé des changements d'états neurologiques et physiologiques significatifs lors de ces évènements théâtraux (comme l'assassinat du bébé mais aussi d'autres évènements bien moins marquants comme de simple déplacements ou regards subtilement mis en scène). Ces changements d'activités neurologiques se sont donc avérés être précisément corrélés aux évènements conçus et travaillés en amont de la représentation (directives précises du metteur en scène relatives au texte dramatique) et portées par le comédien.

L'image scénique perçue par les spectateurs déclenche ainsi un processus qui produit un ensemble dynamique cognitif conduisant le spectateur à co-construire la réalité théâtrale mentalement parallèlement (ou en réaction) aux actions et phénomènes physiques et psychiques produits sur la scène.

L'adhésion du spectateur à la représentation est ainsi intimement liée aux constructions scéniques réalisées par le metteur en scène et l'équipe du spectacle en amont de la représentation. Ces éléments de 
mise en scène vont produire, ponctuellement, chez le spectateur une adhésion à la fiction en parallèle de sa réalité proche. Cette adhésion du spectateur est la résultante d'une activité cognitive complexe et très troublante.

Si le phénomène est simplement observable, qu'en est-il scientifiquement?

Les premiers résultats relatifs aux aires cérébrales activées spécifiquement lors de la mise en action du PAEm ne révèlent rien que l'introspection n'aurait pu permettre d'envisager. En effet, les zones activées lors de l'adhésion du sujet-spectateur aux événements théâtraux sont celles de l'empathie, de la métaphore et de la théorie de l'esprit. Il n'est certes pas question de parler d'une découverte qu'un peu de réflexion ${ }^{5}$ 'aurait permise, mais ces résultats viennent confirmer des intuitions et permettent ainsi d'aller plus loin en imaginant d'autres protocoles expérimentaux.

Il convient de préciser que près de quatre-vingts pour cent des vingt sujets ayant assisté à la représentation théâtrale depuis le scanner (L'enregistreur IRMf), via un dispositif audiovisuel particulier et intégré au spectacle, ont réagi aux événements de mise en scène, et cela, quel que soit leur âge, sexe ou état général (physique et psychologique). En d'autres termes, chaque fois que, dans la représentation, survenait un élément mis en scène (donc prévu a priori) rompant (parfois très subtilement) avec la linéarité du reste de la narration, le sujet-spectateur marquait une réponse hémodynamique ${ }^{6}$ plus ou moins forte qui a permis aux expérimentateurs de mettre au jour les aires activées à ce moment précis.

Un dispositif théâtral a donc été recréé dans un espace du laboratoire (mise au noir, lumières, décors). Pour les vingt sujetsspectateurs retenus pour l'expérience. Cette scène n'était visible que via un écran installé dans un enregistreur IRMf. C'est ainsi qu'ils allaient, successivement, pouvoir assister à une la représentation d'une performance théâtrale de dix minutes retransmise en direct. Pendant ce temps, l'équipe scientifique et technique réalisait des acquisitions neurologiques (IRMf) et cardiologiques (ECG).

Un comédien issu du Théâtre National de Strasbourg, Gaël Chaillat, jouait en direct un extrait de Onysos le Furieux (Gaudet, 2000) de Laurent Gaudé, mis en scène en tenant compte du dispositif technique qui était ainsi intégré au dispositif artistique. De 
cette façon, domaine technique et dimension artistique se mêlaient, brouillant les pistes entre réel et fictif pour le sujet-spectateur dans le but de lui permettre d'adhérer pleinement (comme s'il était dans une salle de spectacle) à la réalité fictive représentée, et ainsi d'entrer de plain-pied au sein du rituel théâtral et de l'émergence de cette réalité.

Les événements déterminés en amont par le metteur en scène (lors des répétitions) serviraient de marqueurs afin de déterminer les moments clefs du spectacle et vérifier si, à ces moments-là, le sujet-spectateur marquait un changement d'état neurobiologique. À ces informations subjectives, s'ajoutaient les données physiologiques (objectives) dégagées par l'ECG.

Pour chaque représentation, dix-sept marqueurs liés à la mise en scène ont été déterminés a priori par le metteur en scène et l'équipe scientifique en tant qu'événements théâtraux à synchroniser avec les images fonctionnelles acquises chez chaque spectateur.

De même, une étude à la première personne est venue compléter ces résultats. Après le spectacle en direct, le sujet-spectateur visionnait le même spectacle qui avait été enregistré en compagnie d'une psychologue et relevait des instants qui l'avaient marqué. La psychologue notait précisément son intérêt subjectif.

Vingt sujets (dix hommes et dix femmes) de vingt à vingt-cinq ans, droitiers, allant au spectacle au moins cinq fois dans l'année ont été sollicités. Installé dans un scanner IRM (deux teslas), chacun visualise, durant un peu plus d'un quart d'heure, la performance théâtrale, le spectacle pensé et construit au sein du dispositif expérimental.

Dès lors, il a été possible aux expérimentateurs d'identifier une réponse neurologique significative, à chaque fois identique et corrélée aux évènements mis en scène, permettant ainsi d'envisager les actions de la mise en scène sur la représentation comme ayant des répercussions importantes. La virtualité de la représentation acquérait ainsi une forme de réalité psychiquement active et repérable scientifiquement. Ces résultats en IRMf étaient complétés par des acquisitions cardiaques et un questionnaire post expérimental qui venait affiner les relevés neuropsychologiques par une approche à la première personne.

La mise en scène aurait donc un rôle majeur et des répercussions importantes dans l'adhésion d'un sujet à une représentation. Le phénomène pourrait sembler tomber sous le sens; il est aujourd'hui avéré. 
Peut alors se poser légitimement, au regard de ces résultats, la question de la manipulation du spectateur par le metteur en scène. Il n'est pas possible à ce stade de notre réflexion d'établir sérieusement une telle problématique et encore moins d'y répondre; en revanche, cette interrogation peut légitimement apparaître aux vues d'un autre élément troublant découvert lors de cette expérience et de l'étude attentive des résultats.

Le point majeur observé dans cette expérimentation est extrêmement curieux et scientifiquement très prometteur. Il concerne tout d'abord la variation de la dynamique cardiaque (diminution) corrélée aux événements théâtraux (mis en scène). L'évolution physiologique du sujet viendrait compléter les éléments neurologiques mis en actions par le PAEm lors de l'émergence de la réalité théâtrale?

Le personnage, ou tout du moins, son inscription représentationnelle, aurait ainsi des répercussions physiologiques fortes sur le sujet-spectateur. Sa présence, liée à la fiction, induit chez le spectateur qui pourtant a conscience de cet état de fiction, des réactions physiologiques corrélées aux changements neurologiques observés, et cela, en particulier lors des évènements mis en scène (conçus en amont de la représentation). Il est entendu que nous parlons ici, dans le cadre de cette expérience, d'une représentation théâtrale. Ainsi, du début de la représentation, il apparaît par convention que le spectateur a accès à une fiction représentée à laquelle il est attendu par l'équipe de conception du spectacle (comédien, metteur en scène) qu'il prête une forme d'existence sans laquelle l'exercice artistique se bornerait à n'offrir qu'un déballage de techniques plus ou moins froid et rébarbatif.

Pour les besoins d'une approche explicative, nous avons dans le présent article simplifié les données neurologiques obtenues et la complexité de la réalité théâtrale qui, bien entendu, ne se limite pas à la seule émergence du personnage.

Dès lors, pour aller plus loin qu'une simple inscription neurologique, cognitive et physiologique du personnage qui pourrait s'entendre aisément par une interprétation du rapport émotionnel qu'entretien le sujet (comédien, spectateur) à la fiction représentée, c'est la découverte, presque par hasard, que ces implications physiologiques matérialisées dans les variations de la dynamique cardiaque semblent correspondre de façon troublante à celles liées à un état du sommeil. 
De fait, l'un des expérimentateurs, Hélène Otzenberger, avait travaillé quelques années auparavant sur l'évolution de la dynamique cardiaque lors du sommeil (Otzenberger; Simon; Gronfier; Brandenberger, 1997, p. 173-176). En observant les relevés cardiologiques ${ }^{7}$ obtenus lors de l'expérience, elle nota des similitudes entre les moments d'adhésion du sujet-spectateur à la représentation et ce qu'elle avait pu observer quelques années plus tôt lors de son étude sur le sommeil. Ces corrélations témoignaient d'un état du sujet qu'il convenait d'éclaircir afin de compléter les relevés en IRMf déjà analysés et interprétés.

Les expérimentateurs ont pu observer que, de façon très significative et pour près de quatre-vingts pour cent des sujets, les activations neurologiques relatives à l'empathie, à la théorie de l'esprit et à la métaphore sont sollicitées par le PAEm. Plus curieux encore, il pourrait apparaître que le spectateur vit cette présence issue de l'émergence du théâtral comme dans un rêve éveillé. Les relevés physiologiques semblent en témoigner. L'adhésion au représenté conduirait-elle alors à une sorte d'oubli de l'espace et du temps physique pour entrer dans ceux de la représentation ou, plus précisément, pour étroitement imbriquer ces deux spatiotemporalités, celle de l'actualité et celle de la fiction, pour en créer une troisième, mitoyenne?

\section{Le Désinvestissement du Soi-spectateur, une Clef?}

Les expérimentateurs ont alors exploré cette piste marquée physiologiquement en la complétant par les relevés neurologiques concomitants. Ils ont alors mis au jour une activation, ou plus précisément une désactivation cérébrale, qui pourrait expliquer l'émergence de la réalité spatiotemporelle représentationnelle. Cette découverte vient compléter les trois activations cérébrales déjà relevées ainsi que la diminution de la dynamique cardiaque du sujet afin que le principe d'adhésion émergentiste s'active et engage le sujet-percevant-participant à accepter que la représentation ait une forme de réalité.

Concrètement, les relevés expérimentaux ont montré que les corrélats physiologiques associés aux instants d'adhésion du spectateur à la réalité théâtrale étaient concomitants à une diminution de la variabilité du rythme cardiaque. Cela se traduit chez le spectateur par une absence d'engagement des régions cérébrales impliquées dans la référence à soi (l'autoréférence). Ces états observés lors de la mise 
en action du PAEm face à une représentation théâtrale sont comparables aux états hypnotiques caractérisés par la dissociation entre l'expérience mentale en cours et l'expérience physique immédiate.

Cet état physique et cognitif est probablement la clef de voûte du système représentationnel théâtral qui permettra aux présences fictives représentées sur la scène d'accéder à une forme de présence qui pourra affecter émotionnellement le spectateur. De fait, le théâtral via le PAEm (et en particulier l'état hypnotique ponctuel observé) est la réunion de deux phénomènes a priori fort lointains: l'actualité et la virtualité. L'état hypnotique est peut-être le ciment primordial entre ces deux états.

Un autre phénomène vient fort probablement renforcer l'adhésion et compléter la compréhension du phénomène d'émergence d'une réalité: il s'agit de l'interindividualité.

\section{L'Interindividualité: un substrat du théâtral}

La virtualité du personnage, a fortiori lorsqu'un spectacle engage une représentation au second degré ${ }^{8}$, s'inscrit dans la coprésence acteur/ spectateur (même à distance) pour faire émerger une spatiotemporalité particulière, celle de la représentation. Cette réalité mi-actuelle, mi-virtuelle à la fois est l'un des enjeux fondamentaux du théâtral auquel le spectateur sera conduit à donner existence par sa bonne foi, par son adhésion, afin qu'émerge la réalité théâtrale pleine et entière. Le comédien/personnage sera le pivot, l'athanor, qui cristallisera en lui ce double état de réalité, simultanément actuel et virtuel. Cet état mitoyen fait écho à la réflexion de Coleridge sur la "[...] suspension consentie de l'incrédulité ${ }^{9} »(1817$, n.p.) comme condition d'entrée dans la fiction théâtrale et qui pose ainsi l'un des fondements majeurs du travail neuropsychologique sur le principe d'adhésion émergentiste que nous effectuons aujourd'hui.

L'un des enjeux de l'expérience théâtre et neurosciences cognitives de Strasbourg a été de recréer cette coprésence essentielle à l'émergence du théâtral. Il s'avérait donc fondamental, dès le montage de cette expérience, d'intégrer le dispositif technique au dispositif artistique. Plus précisément, le travail majeur de la mise en scène devait jouer avec le lourd environnement médical, l'intégrer dans la représentation et ainsi brouiller les pistes entre réel et fictif en jouant de la présence de l'acteur afin de rendre une forme de présence active de la fiction au sujet- 
spectateur. Dès lors, au cours des répétitions, une attention particulière a été accordée au bruit (qui est de l'ordre de quatre-vingts-quatre-vingt dixdB lors des acquisitions en IRMf), afin de l'intégrer au temps de jeu et de déplacement du comédien pour en faire un partenaire, un outil de mise en scène et non un élément contre lequel il fallait lutter, ce qui se serait révélé vain ou, tout du moins, fort difficile compte tenu du volume de décibels produit par la machine.

Dès son entrée dans la salle du scanner, le sujet est confronté à un individu (le comédien/personnage) installé sur le lit sur lequel lui-même doit prendre place pour entrer dans la machine. Autour d'eux, médecins et ingénieurs s'affairent (ou font mine de s'affairer pour renforcer l'effet dramatique). L'acteur accueille le sujet avec des phrases qui sont déjà le texte de la pièce. Il se lève et l'invite à prendre sa place: «[...] tu n'as pas besoin de prétexte pour venir t'asseoir à côté de moi» (Gaudé, 2000, p. 9). Un ingénieur de l'équipe dispose le sujet correctement, lui donne les consignes de sécurité et quelques informations pour le bon déroulement de l'expérience. Pendant ce temps, l'acteur continue, ponctuellement, à livrer son texte tout en l'intégrant aux directives d'usage. Ainsi, lorsque l'ingénieur dit selon les recommandations techniques et de sécurités utilisées en imagerie médicale: "[...] si vous avez un problème appuyez sur la poire», l'acteur intervient et, en tant que personnage, ajoute: «[...] n'aies pas peur» (Gaudé, 2000, p. 10). La coprésence acteur/spectateur fondamentale à l'élaboration du théâtral pouvait trouver là une forme de réinvestissement. Les enregistrements neurologiques et physiologiques débutaient alors. La représentation se mettait en place, le principe d'adhésion émergentiste pouvait s'activer, la réalité théâtrale émerger et le spectateur être ému.

\section{Vers une Utilisation des Résultats Obtenus et la Stabilisation d'un Concept}

Les résultats scientifiques obtenus lors de ces enregistrements ainsi que les perspectives ouvertes sont d'ores et déjà explorés dans d'autres champs d'études comme, par exemple, en neuro-éducation.

Une vaste étude théorique et pratique, pilotée par Yannick Bressan et le Dr. Safouane Hamdi (Hôpital de Toulouse), sur le lien principe d'adhésion, transmission et mémorisation des savoirs est en cours (Bressan; Hamdi, 2013). Un travail en psycho-oncologie est, 
par ailleurs, réalisé par Yannick Bressan à l'université de psychologie clinique de Strasbourg.

Il reste qu'une des implications phénoménologiques fondamentales mise en évidence dans cette expérience est le lien indéfectible qui unit la représentation et le sujet percevant afin de faire émerger une réalité (ici théâtrale), et cela, à travers le principe d'adhésion émergentiste.

Ce phénomène psycho-cognitif serait ainsi, dans cette perspective, un méta phénomène au fondement cognitif de l'ensemble de notre système représentationnel.

De fait, sans adhésion à des propos ou à une réalité (politique, religieuse, commerciale, communicationnelle, éducationnelle), que reste-t-il de cette réalité? À l'image de la représentation théâtrale face à laquelle le sujet ne prêterait pas une forme existentielle et qui ne resterait qu'un simple exercice technique, sans adhésion que reste-t-il de notre réalité ? Au-delà de la simple adhésion qui est un phénomène bien connu en psychologie (Orfali, 2011), il apparait un concept qui l'englobe et le dépasse tout en y étant très lié: l'adhésion émergentiste. Celle-ci n'est pas le seul intérêt d'un sujet pour un phénomène lié à sa réalité proche mais, à partir de l'intérêt et l'engagement que suppose l'adhésion et via les activations cérébrales et physiologiques observées, peut ponctuellement émerger avec force le théâtral. Cette fiction représentée à laquelle assiste activement le spectateur peut bouleverser sa propre lecture du monde suite aux choix du metteur en scène portés par le comédien et l'univers théâtral(isé). La préhension et l'intégration du spectateur au sein du monde peuvent ainsi s'en trouver (plus ou moins) bouleversées, remodelées, renforcées.

L'outil en psychologie qu'est le principe d'adhésion émergentiste peut ainsi s'avérer fondamental pour de nombreuses recherches théoriques et pratiques.

Outre les enjeux qui peuvent sembler tomber sous le sens, esthétiques et artistiques d'une telle question, il est très prometteur et stimulant pour la recherche scientifique et en sciences humaines, d'envisager le théâtre, sa puissance symbolique et cognitive comme une source expérimentale féconde. C'est ainsi, peut-être, avant tout ce qu'a démontré ce travail: la force de l'interdisciplinarité, la pertinence de l'approche systémique et l'immense potentiel scientifique du théâtral. 


\section{Notes}

${ }^{1}$ Étude réalisée en 2007-2008 au Laboratoire d'Imagerie et Neurosciences Cognitives, CNRS, en collaboration avec le Théâtre National de Strasbourg. Cette expérience fut conçue et dirigée par Bressan qui signa, de plus, la mise en scène utilisée dans le protocole expérimental. Cette étude fut, par ailleurs, suivie d'un point de vue médical par la neurologue Metz-Lutz. Cette expérience est présentée et analysée en détail dans Le Théâtral comme Lieu d'Expérience des Neurosciences Cognitives (Bressan, 2013).

${ }^{2}$ La Systémique (Durand, 2013).

${ }^{3}$ La notion philosophique de par-soi, empruntée à Spinoza (1999, p. 278), recouvre ici un sens différent. L'acception que nous donnons à ce terme engage une dimension émergentiste (et non religieuse comme chez Spinoza) et complète quelque peu la traduction de l'expression latine per se qui signifie ce qui existe en fonction de son essence. Ici, le par-soi est le processus selon lequel le sujet percevant, à partir des éléments physiques absolus qui lui sont donnés à percevoir, va faire émerger, par sa perception, sa propre réalité.

${ }^{4}$ Le comme si stanislavskien.

${ }^{5}$ Au sujet de la métaphore, Aristote l'envisageait déjà dans La Poétique!

${ }^{6}$ Variation du flux sanguin dans le cerveau. Phénomène permettant l'emploi de la technique des IRMf (images par résonances magnétiques fonctionnelles).

${ }^{7}$ Les sujets-spectateurs assistant à la représentation en direct depuis le scanner étaient aussi équipés d'enregistreurs cardiaques, voir, pour plus de précisions sur cette expérience et l'analyse des premiers résultats obtenus Le Théâtre comme Lieu d'Expérience des Neurosciences Cognitives, à la Recherche du Principe d'Adhésion (Bressan, 2013).

${ }^{8}$ Représentation retransmise en direct sur un écran, en utilisant les nouvelles technologies telles qu'Internet ou les écrans vidéo, pour être accessible aux spectateurs. Dans le cadre de l'expérience de mise en évidence du principe d'adhésion, le recours à la re-représentation (ou représentation au second degré) a été une obligation technique afin que le sujet-spectateur à l'intérieur de l'enregistreur IRMf puisse assister en direct au spectacle.

${ }^{9}$ En anglais au original: “[...] it was agreed, that my endeavours should be directed to persons and characters supernatural, or at least romantic, yet so as to transfer from our inward nature a human interest and a semblance of truth sufficient to procure for these shadows of imagination that 'willing suspension of disbelief for the moment, which constitutes poetic faith" (Coleridge, 1817, n.p.).

\section{Références}

ARTAUD, Antonin. Le Théâtre et son Double. Paris: Gallimard, 2001. (Collection Folio Essais.)

BRESSAN, Yannick. Le Théâtre comme Lieu d'Expérience des Neurosciences Cognitives. Paris: L'Harmattan, 2013. 
BRESSAN, Yannick; HAMDI, Safouane. Du Théâtre à l'Amphithéâtre: pour une extension du concept d'adhésion à la neuroscience éducationnelle. Revue de l'Association for Research in Neuroeducation, Association pour la Recherche en Neuroéducation, v. 2, n. 1, p. 1-15, déc. 2013.

COLERIDGE, Samuel Taylor. Biographia Literaria. [S.1.]: Project Gutenberg, 1817. Disponible sur: <http://www.gutenberg.org/>. Consultée sur: [n.d.].

DURAND, Daniel. La Systémique. Paris: Presses Universitaires de France, 2013. (Collection Que Sais-Je?).

GAUDÉ, Laurent. Onysos le Furieux. Paris: Actes Sud, 2000.

JAMES, William. La Volonté de Croire. Paris: Les Empêcheurs de Penser en Rond/Seuil, 2005.

MAUSS, Marcel. Sociologie et Anthropologie. Paris: P.U.F, 1983 [1950]. (Collection Quadrige.)

ORFALI, Birgitta. L’Adhésion. Bruxelles: De Boeck, 2011.

OTZENBERGER, Hélène et al. Temporal Relationship between Dynamic Heart Rate Variability and Electroencephalographic Activity during Sleep in Man. Neuroscience Letters, [s.1.], Elsevier, v. 229, p. 173-176, 1997.

Yannick Bressan est chercheur à Université de Psychologie de Strasbourg. Il est docteur en sciences humaines (Paris X Nanterre). Il enseigne depuis plus de dix ans les arts visuels et les nouvelles technologies. Il a mis en scène en 1999 l'une des premières pièces de théâtre interactives présentée exclusivement en direct sur Internet, posant ainsi la question de la représentation au second degré.

E-mail: bressan30@hotmail.com 